Severity of frailty and the outcome of exercise intervention among participants with Alzheimer disease : A sub-group analysis of a randomized controlled trial

\title{
Perttilä, Niko
}

\section{6-04}

Perttilä , N , Ohman , H , Strandberg , T E , Kautiainen , H , Raivio , M , Laakkonen , M -L , Savikko , N , Tilvis , R S \& Pitkala , K H 2016 , ' Severity of frailty and the outcome of exercise intervention among participants with Alzheimer disease : A sub-group analysis of a randomized controlled trial ' , European Geriatric Medicine , vol. 7 , no. 2 , pp. 117-121 . https://doi.org/10.1016/j.eurs

http://hdl.handle.net/10138/223939

https://doi.org/10.1016/j.eurger.2015.12.014

publishedVersion

Downloaded from Helda, University of Helsinki institutional repository.

This is an electronic reprint of the original article.

This reprint may differ from the original in pagination and typographic detail.

Please cite the original version. 
Research paper

\title{
Severity of frailty and the outcome of exercise intervention among participants with Alzheimer disease: A sub-group analysis of a randomized controlled trial
}

\author{
N.M. Perttila ${ }^{\text {a,* }}$, H. Öhman ${ }^{\text {a,b }}$, T.E. Strandberg ${ }^{c, d}$, H. Kautiainen ${ }^{\text {a }}$, M. Raivio ${ }^{\text {, }}$ \\ M.-L. Laakkonen ${ }^{\mathrm{a}, \mathrm{b}}$, N. Savikko ${ }^{\mathrm{c}, \mathrm{e}}$, R.S. Tilvis ${ }^{\mathrm{d}}$, K.H. Pitkala ${ }^{\mathrm{a}}$ \\ a University of Helsinki, Department of General Practice and Unit of Primary Health care, Helsinki University Central Hospital, Tukholmankatu 8 B, PL20, \\ 00014 Helsingin yliopisto, Helsinki, Finland \\ ${ }^{\mathrm{b}}$ City of Helsinki, Hospital, Rehabilitation and Care services, Helsinki, Finland \\ ${ }^{\mathrm{c}}$ University of Helsinki, Department of Internal Medicine and Geriatrics, Finland, and Clinics of Internal Medicine and Geriatrics, Helsinki University Central \\ Hospital, Helsinki, Finland \\ ${ }^{\mathrm{d}}$ University of Oulu, Department of Public Health Science and General Practice, Oulu University Hospital, Oulu, Finland \\ ${ }^{\text {e }}$ City of Espoo, Home Care, Espoo, Finland
}

\section{A R T I C L E I N F O}

\section{Article history:}

Received 5 October 2015

Accepted 26 December 2015

Available online 21 January 2016

\section{Keywords:}

Frailty

Exercise

Alzheimer disease

Falls

Functional performance

\begin{abstract}
A B S T R A C T
Introduction: To investigate how frailty status affects the outcome of exercise intervention among homedwelling participants with Alzheimer disease (AD).

Methods: This is a sub-group analysis of a randomized controlled trial. In this trial, home-dwelling participants with AD received either home-based or group-based exercise twice a week for one year $(n=129)$; the control group received normal care $(n=65)$. Both the intervention and control group were subdivided into two groups according to modified Fried criteria: prefrail (0-1 criteria) and advanced frailty (2-5 criteria). The Functional Independence Measure (FIM) and number of falls per person-years served as outcome measures.

Results: Whereas there was no significant difference in FIM between the prefrail intervention (PRI) and control (PRC) groups at 3 or 6 months, the PRI group deteriorated significantly slower at 12 months ( -6.6 [95\% CI -8.6 to -4.5$]$ for PRI and $-11.1[95 \% \mathrm{CI}-13.9$ to -8.3 ] for PRC; $P=0.010$ ). Similarly, there was no significant difference between the advanced frailty intervention (AFI) and control (AFC) groups at 3 months, but the difference became significant at 6 months $(-8.1$ [95\% CI -11.1 to -5.2$]$ for AFI and -15.5 [95\% CI -20.0 to -11.1 ] for AFC; $P=0.007)$ and at 12 months $(-8.9$ [95\% CI -11.9 to -5.9 ] for AFI and -15.3 [ $95 \% \mathrm{CI}-20.2$ to -10.3 ] for AFC; $P=0.031$ ). There was also a significant difference in the number of falls in favor of PRI and AFI groups compared to their respective control groups.

Conclusion: A long-term exercise intervention benefited people with $\mathrm{AD}$ regardless of their stage of frailty.

Trial registration: : ACTRN12608000037303.
\end{abstract}

(c) 2016 Elsevier Masson SAS and European Union Geriatric Medicine Society. All rights reserved.

\section{Introduction}

The term frailty is used to describe older people whose health has deteriorated, without being a direct consequence of one or multiple diseases [1]. Older people with frailty are vulnerable to multiple complications [1,2]. Researchers have proposed several definitions for frailty, but the Fried criteria [3] are the most

\footnotetext{
* Corresponding author. Tel.: +358504136188.

E-mail address: niko.perttila@helsinki.fi (N.M. Perttila).
}

frequently used [4]. Although Fried criteria based only on physical conditions have come under criticism for failing to take into consideration biological, psychological and social dimensions [5], limiting the definition to individual's physical dimension simplifies research and enables comparison of different studies [5].

Frailty is known to have poor prognosis [2], as it leads to disabilities, complications and increased risk of death [1$3,6,7]$. The prevention and treatment of frailty has seen extensive research [8]. Several systematic reviews suggest that exercise benefits frail people by improving their balance, gait speed and functioning [9-11]. Most benefits derive from diverse, long-term 
and intense exercise training [11]. Diverse strength, balance and endurance training seems most effective in decreasing the number of falls and in improving gait and physical ability to function among physically frail older people [12]. Some researchers have suggested that exercise intervention may benefit prefrail people more than frail people [13]. However, a recent study found that frail participants benefited the most from physical activity intervention [14]. Other researchers have argued that physical activity intervention may even reduce frailty [14,15].

A number of studies have shown that frailty increases the risk for cognitive decline, and that cognitive impairments increase the risk for frailty [16]. Frailty and dementia share similar features: both conditions increase with aging and share the same etiological factors, such as smoking, low physical activity, obesity and depression [17]. Malnutrition and weight loss are common problems in both AD [18] and frailty [12]. The loss of lean mass leads to an increased risk for sarcopenia in AD [19], which is associated with frailty [20]. A decrease in muscle mass and strength predisposes older adults to both frailty and falls [3].

Although people with dementia seem to benefit from exercise interventions [21-23], to our knowledge, no intervention studies have explored how the frailty status of people with Alzheimer disease modifies the effectiveness of exercise. This study is a subgroup analysis of a randomized controlled trial, which investigated the costs and effects of an exercise intervention on participants with Alzheimer disease [23]. The aim of this study was to investigate how the frailty status of participants with Alzheimer disease modifies the outcome of an intense and long-term exercise intervention with respect to their physical functioning and falls.

\section{Methods}

The original randomized, controlled FINALEX study comprised two active intervention arms: home-based and group-based exercise intervention, both of which consisted of similar exercise components in approximately one-hour sessions held twice weekly for one year [23]. For this sub-analysis, we merged both exercise groups into one intervention group. Physiotherapists supervised both types of interventions. For the sub-group analysis, we subdivided both the control group and the intervention group into two groups according to their frailty status: a prefrail intervention group (PRI) and a prefrail control group (PRC) meeting $0-1$ of the five Fried criteria [3], and an advanced frailty intervention group (AFI) and an advanced frailty control group (AFC) meeting $2-5$ of the five Fried criteria.

\subsection{Participants}

In 2008, the Social Insurance Institution of Finland used its drug reimbursement register to recruit Alzheimer patients living with a spouse in the cities of Helsinki, Espoo or Vantaa $(n=1264)$. Altogether 497 persons expressed an interest in participating. Study nurses managed to contact 390 persons, 84 of which did not wish to participate and 96 did not fulfil the inclusion criteria, which were:

- speaking Finnish language;

- living with a spouse at home;

- living in Helsinki, Espoo or Vantaa;

- $\geq 65$ years, retired;

- no diagnosed terminal disease or difficult hemiplegia;

- the ability to walk independently with or without a mobility aid.

All participants had to have at least one of the following signs of possible frailty: $\geq 1$ falls during the previous 12 months, unintentional weight loss, or decreased walking speed. Therefore, some participants may have had only a fall as a sign of frailty, and so did not meet any of the modified Fried criteria. A total of 210 patients met our inclusion criteria and participated in the study.

The Ethics Committee of the Helsinki University Central Hospital approved the study, and all patients provided informed consent. Spouses provided informed consent for patients with reduced judgment capacity.

\subsection{Clinical measures}

We collected data on demographic factors (age, sex, education) at baseline. The Mini Nutritional Assessment (MNA) served to assess the participants' nutritional status [24], and their medical records served to confirm their medication and comorbidities; we then calculated the Charlson comorbidity index [25]. The Clinical Dementia Rating (CDR) scale [26] and the Mini Mental State Examination (MMSE) [27] served to evaluate cognitive status, and the Functional Independence Measure (FIM) [28] to evaluate physical functioning.

The participants in either intervention arms and the participants in the control group were divided into two groups according to their clinical stage of frailty as determined by the modified Fried criteria [3]. In this study, the five frailty criteria were:

- unintentional weight loss - the spouse was asked (yes/no);

- exhaustion - based on item retrieved from Cornell Scale for Depression in Dementia [29]: "Lack of energy: fatigues easily, unable to sustain activities";

- low physical activity - the question inquired whether the participant exercised in their leisure time (yes/no);

- slowness - based on the walking speed in the Short Physical Performance Battery test (SPPB) $(<0.85 \mathrm{~m} / \mathrm{s})$ [30];

- weakness - based on the grip strength in the SPPB test [30].

Patients meeting none or only one of the above criteria were classified into the prefrail groups (PRI and PRC), and patients meeting 2-5 criteria were classified into the advanced frailty groups (AFI and AFC).

We examined the effects of an exercise intervention on physical functioning during a one-year follow-up among Alzheimer patients with various stages of frailty in both groups (PRI vs. PRC and AFI vs. AFC) separately. We assessed the effects of the intervention as changes in FIM [28]. We assessed FIM values at baseline, 3, 6, and 12 months based on the caregiver's evaluation of the patient's performance at home. FIM assesses both physical (13 questions) and cognitive functioning ( 5 questions). Each item is evaluated on a 1 - to 7 -point scale ( 1 = the greatest need for help; 7 = the least). Total scores fall between 18 and 126; the lower the score, the greater the need for help [28].

\subsection{Interventions}

A detailed description of the intervention has been published previously [22]. Patient safety was ensured by a geriatrician who assessed each participant's health status starting in the intervention group.

The home exercise group received a tailored exercise intervention at patients' homes for two one-hour sessions per week for one year. A physiotherapist administered the intervention, which was individually tailored to improve every-day skills while taking into account patients' needs. The group-based exercise intervention also trained for about one hour twice a week for one year. The visits to adult daycare centers lasted for four hours, of which the individual training time lasted one hour. Ten patients and two 
physiotherapists participated at a time. Both groups participated in strength, balance, endurance and multi-tasking training.

Controls received normal community care and were allowed to receive rehabilitation in the public health care system.

All patients and their spousal caregivers in both the intervention and control groups received advice on exercise and nutrition. In addition, the use of vitamin D supplements $(20 \mu \mathrm{g} / \mathrm{d})$ was recommended to all.

\subsection{Statistical analysis}

The data appear as counts and percentages or as means with standard deviations. The $t$-test, the Mann-Whitney U-test, or the $\mathrm{Chi}^{2}$ test served to test the differences between the groups at baseline. We used generalized linear mixed-models with unstructured correlation structure to analyze repeated measures. Fixed effects were group, time, and group-time interaction. We estimated and compared the incidence rate of falls using Poisson regression models or negative binomial regression models when appropriate. We used the Lagrange multiplier test to test the assumptions of over-dispersion in the Poisson model.

\section{Results}

Eleven participants declined to participate and five died immediately after randomization. The number of participants included in this sub-group analysis was 194 (111 participants in the prefrail groups and 83 participants in the advanced frailty groups).

Table 1 shows the participants' baseline characteristics. In the prefrail group (presenting with 0-1 Fried criteria), a larger percentage of the participants in the intervention group had slow gait speed than in the control group. In the prefrail group, the Charlson index revealed more comorbidities among participants in

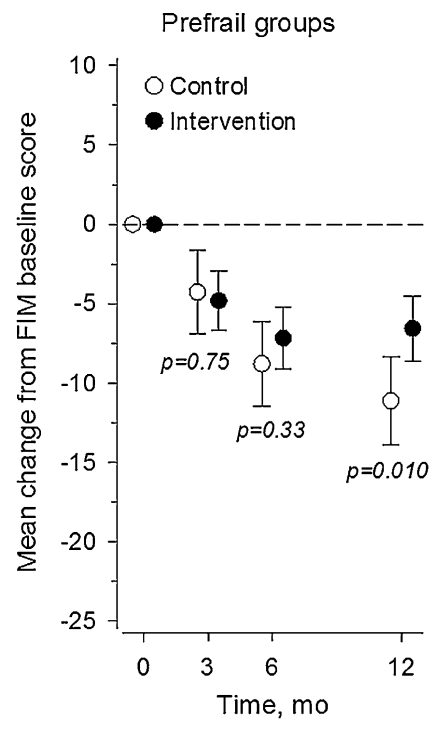

Advanced frailty groups

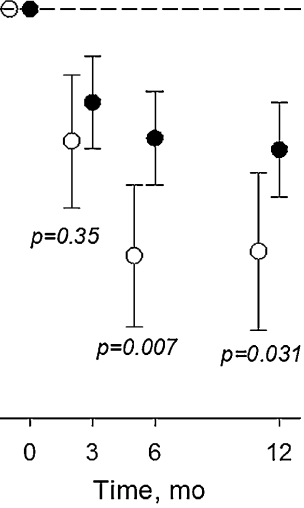

Fig. 1. The mean changes from the Functional Independence Measure (FIM) baseline scores in the prefrail intervention and control groups (left panel) and advanced frailty intervention and control groups (right panel). The analyses were adjusted for age, gender and Charlson comorbidity index.

the control group. Neither group (prefrail, advanced frailty) in the intervention and control arms showed any significant differences in other baseline characteristics.

\subsection{Effects of the intervention on physical functioning}

The left panel on Fig. 1 presents the changes in physical functioning in the patient group in the prefrail stage (0-1 frailty criteria) during the 12-month follow-up. FIM scores showed a

Table 1

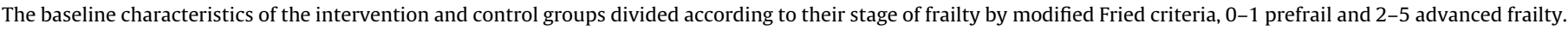

\begin{tabular}{|c|c|c|c|c|c|c|}
\hline & \multicolumn{6}{|l|}{ Frailty criteria } \\
\hline & \multicolumn{3}{|l|}{ Prefrail $(0-1)$} & \multicolumn{3}{|c|}{ Advanced frailty (2-5) } \\
\hline & $\begin{array}{l}\text { Intervention } \\
n=73\end{array}$ & $\begin{array}{l}\text { Control } \\
n=38\end{array}$ & $P$-value ${ }^{\mathrm{a}}$ & $\begin{array}{l}\text { Intervention } \\
n=56\end{array}$ & $\begin{array}{l}\text { Control } \\
n=27\end{array}$ & $P$-value ${ }^{\mathrm{a}}$ \\
\hline Males, $n(\%)$ & $53(73)$ & $25(66)$ & 0.46 & $27(48)$ & $14(52)$ & 0.76 \\
\hline Age, mean (SD) & $77.8(5.2)$ & $76.8(5.3)$ & 0.40 & $78.1(5.6)$ & $80.1(4.7)$ & 0.072 \\
\hline Education < $8 \mathrm{y}, n(\%)$ & $26(36)$ & $14(37)$ & 0.90 & $22(39)$ & $15(56)$ & 0.16 \\
\hline CDR, $n(\%)$ & & & 0.13 & & & 0.66 \\
\hline $0.5-1$ & $29(40)$ & $17(45)$ & & $15(27)$ & $5(18)$ & \\
\hline 2 & $33(45)$ & $20(53)$ & & $28(50)$ & $14(52)$ & \\
\hline 3 & $11(15)$ & $1(3)$ & & $13(23)$ & $8(30)$ & \\
\hline BMI, mean (SD) & $25.5(3.1)$ & $25.1(3.3)$ & 0.50 & $26.2(3.8)$ & $24.8(4.7)$ & 0.14 \\
\hline MNA, $n(\%)$ & & & 0.76 & & & 0.14 \\
\hline$<17 \mathrm{p}$, malnourished & $0(0)$ & $0(0)$ & & $1(2)$ & $3(11)$ & \\
\hline $\begin{array}{l}17-23.5 \mathrm{p} \text {, at-risk } \\
\text { for malnutrition }\end{array}$ & $52(71)$ & $26(68)$ & & $42(75)$ & $20(74)$ & \\
\hline$>23.5$ p, well-nourished & $21(29)$ & $12(32)$ & & $13(23)$ & $4(15)$ & \\
\hline \multicolumn{7}{|l|}{ Fried criteria } \\
\hline Weak grip strength, $n(\%)$ & $3(4)$ & $5(13)$ & 0.080 & $33(59)$ & $16(59)$ & 0.98 \\
\hline Weight loss, $n(\%)$ & $2(3)$ & $1(3)$ & 0.97 & $18(32)$ & $7(26)$ & 0.56 \\
\hline Slow gait speed, $n(\%)$ & $27(37)$ & $7(18)$ & 0.044 & $47(84)$ & $23(85)$ & 0.88 \\
\hline Exhaustion, $n(\%)$ & $1(1)$ & $2(5)$ & 0.23 & $8(14)$ & $6(22)$ & 0.37 \\
\hline Low physical activity, $n(\%)$ & $8(11)$ & $5(13)$ & 0.73 & $30(54)$ & $18(67)$ & 0.26 \\
\hline Charlson, mean (SD) & $2.6(1.8)$ & $3.0(1.5)$ & 0.046 & $2.5(1.7)$ & $2.9(1.9)$ & 0.39 \\
\hline Medication, mean (SD) & $6.1(3.1)$ & $6.2(2.4)$ & 0.53 & $7.0(4.0)$ & $7.0(3.7)$ & 0.84 \\
\hline MMSE, mean (SD) & $19.2(6.1)$ & $19.6(5.2)$ & 0.96 & $16.9(7.0)$ & $15.6(6.4)$ & 0.30 \\
\hline \multicolumn{7}{|l|}{ FIM, mean (SD) } \\
\hline Total & $93.0(16.8)$ & $95.5(13.7)$ & 0.44 & 81.7 (19.6) & 76.5 (17.3) & 0.22 \\
\hline Motor & $74.5(12.6)$ & $76.0(10.8)$ & 0.65 & $64.9(16.6)$ & $61.8(13.3)$ & 0.26 \\
\hline Cognitive & $18.5(5.7)$ & $19.5(4.4)$ & 0.31 & $17.0(6.0)$ & $14.7(5.7)$ & 0.095 \\
\hline
\end{tabular}

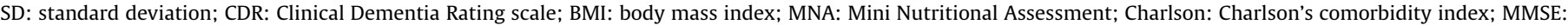
Mini Mental State Examination; FIM: Functional Independence Measure.

a Differences between groups were tested with the $t$-test or the Mann-Whitney U-test, and the Chi ${ }^{2}$ or Fischer's exact test when appropriate. 
decline in physical functioning in both the control and intervention groups. However, the rate of deterioration was slower in the intervention group than in the control group. The mean difference between the groups was significant at 12 months (FIM change, -6.6 [95\% CI: -8.6 to -4.5$]$ in the intervention group and -11.1 [95\% CI: -13.9 to -8.3 ] in the control group; $P=0.010$ adjusted for age, sex and comorbidities).

The right panel on Fig. 1 presents the changes in physical functioning in the advanced frailty groups (2-5 frailty criteria). Although physical functioning deteriorated in both groups, the rate of decline was significantly slower in the AFI group than in the AFC group. The mean difference between the groups was significant at 6 months (FIM change, -8.1 [95\% CI: -11.1 to -5.2$]$ in the intervention group and -15.5 [95\% CI: -20.0 to -11.1 ] in the control group; $P=0.007$ adjusted for age, sex and comorbidities) and at 12 months (FIM change, -8.9 [95\% CI: -11.9 to -5.9$]$ in the intervention group and -15.3 [95\% CI: -20.2 to -10.3 ] in the control group; $P=0.031$ adjusted for age, sex and comorbidities).

\subsection{Effects of the intervention on falls}

Fig. 2 presents the effects of the exercise intervention on falls during the 12-month intervention period. In the prefrail groups ( 0 1 frailty criteria, left panel on Fig. 2), the participants in the intervention group had a significantly lower rate of falls than those in the control group ( 1.14 falls/person per year [ $95 \% \mathrm{CI}$ : 0.90 to 1.43 ] and 1.82 falls/person per year [95\% CI: 1.40 to 2.32 ], respectively). The incidence rate ratio (IRR) was 0.63 [95\% CI: 0.45 to 0.89 ]; $P=0.008$ adjusted for sex, age and comorbidities.

In the advanced frailty groups (2-5 frailty criteria, right panel on Fig. 2), the average rate of falls was also significantly lower in the intervention group than in the control group ( 2.15 falls/person per year [ $95 \% \mathrm{CI}: 1.76$ to 2.59$]$ and 5.32 falls/person per year [95\% CI: 4.36 to 6.44 ], respectively). The IRR was 0.43 [95\% CI: 0.33 to 0.57]; $P<0.001$ adjusted for sex, age and comorbidities.

\section{Discussion}

These sub-group analyses of an exercise trial suggest that an intensive and long-term exercise intervention may significantly slow the deterioration of physical functioning in persons with Alzheimer's disease at different stages of frailty. This effect was significant in both prefrail and advanced frailty persons with AD.

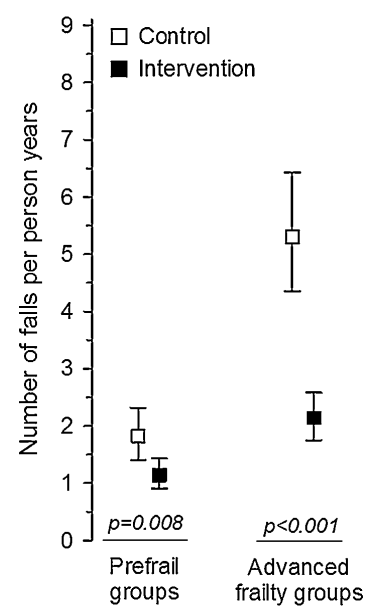

Fig. 2. Number of falls per person-years in the prefrail intervention and control groups (left panel) and the advanced frailty intervention and control groups (right panel). The analyses were adjusted for age, gender and Charlson comorbidity index.
Moreover, in both prefrail and advanced frailty adults, the intervention significantly reduced the number of falls.

This study has several strengths. To our knowledge, this is the first study to examine the effects of physical exercise in $A D$ patients at different stages of frailty. The definition of frailty stage is based on the Fried criteria [3], which predict important endpoints such as disabilities and death [3]. Although this is a sub-group analysis of the primary randomized trial, both the prefrail groups as well as the advanced frailty groups were well comparable at baseline. The intervention was well coordinated, and a physiotherapist supervised each training session, which was also frequent (twice a week) and long-term (52 weeks). The intervention, implemented by a single professional group (physiotherapists) in primary care, did not increase the total costs of health and social services [23].

This study also has several limitations. This is a post-hoc analysis of a randomized controlled trial. Therefore, the groups compared are not original randomized groups. In the advanced frailty groups there was almost significant difference between the intervention and control groups concerning age. However, all analyses were adjusted for age, gender and comorbidities. All the participants were motivated volunteers living in with their spousal caregivers in their homes in the community, and all were Caucasian. Generalizing of these results to other populations should be made with caution. The small sample size decreases the power of the study. Therefore, we could not divide the groups into traditional robust, prefrail and frail groups [3]. Nor was the study blinded. However, the study nurses collecting the data were uninformed about the exact intervention and primary outcome measures, and were not researchers in the study. Moreover, the FIM [28] was assessed based on the spousal caregivers' evaluations and they were also unaware of the study hypothesis. The control patients received high-quality community care, which presumably decreases the differences between the groups.

Some researchers have suggested that exercise is beneficial in frailty by improving physical functioning [9-11], which is in line with our finding. Other researchers have suggested that prefrail people could benefit more from an exercise intervention than frail people [13]. In contrast to this finding, our advanced frailty group seemed to benefit even earlier from the exercise intervention than the prefrail group. However, in line with our findings, a recent post-hoc analysis of the LIFE-P study suggested that the frail participants benefited more from the exercise intervention than the non-frail participants did [14]. In our study, both the prefrail and advanced frailty intervention groups deteriorated significantly more slowly and experienced significantly fewer falls than did the prefrail and advanced frailty control groups. Reducing the number of falls through an exercise intervention is in accordance with the results of previous studies [12]. Both intervention groups probably saw such benefits as a result of a frequent, diverse and long-term exercise intervention, which also yielded the most benefits in previous studies [11].

Frailty status logically and consistently associated with gender, stage of dementia, and physical functioning. The participants in our prefrail and advanced frailty groups shared similar baseline characteristics to those in previous studies $[3,16,18]$. The advanced frailty groups had more women and used more medication than did the prefrail groups. In addition, the stage of dementia was more advanced in the advanced frailty groups than in the prefrail groups, which is in accordance with the findings of previous studies that frailty becomes more prevalent when dementia advances [16]. Malnutrition is common among frail people [12], and our study had larger proportions of wellnourished participants in the prefrail groups than in the advanced frailty groups. 


\section{Conclusion}

These results indicate that persons with Alzheimer disease independent of their stage of frailty could benefit from a frequent and long-term exercise training program. Both prefrail and advanced frailty $\mathrm{AD}$ patients may benefit in respect to physical functioning and falls.

\section{Ethical statement}

The Ethics Committee of the Helsinki University Central Hospital approved the study, and all patients provided informed consent. Spouses provided informed consent for patients with reduced judgment capacity. All procedures of the study were carried out according to Helsinki Declaration.

\section{Disclosure of interest}

The authors declare that they have no competing interest.

\section{Acknowledgments}

This study was supported by the Social Insurance Institution of Finland, the Central Union for the Welfare of the Aged, the Sohlberg Foundation, and the King Gustaf V and Queen Victoria Foundation. The sponsors had no role in the study design, data analysis, or interpretation of the results, nor did they have a role in writing the report or in the decision to submit it for publication. The authors were independent researchers unassociated with the sponsors.

\section{References}

[1] Fulop T, Larbi A, Witkowski JM, McElhaney J, Loeb M, Mitnitski A, et al. Aging, frailty and age-related diseases. Biogerontology 2010;11(5):547-63.

[2] Rockwood K, Mitnitski A. Frailty in relation to the accumulation of deficits. J Gerontol A Biol Sci Med Sci 2007;62(7):722-7.

[3] Fried LP, Tangen CM, Walston J, Newman AB, Hirsch C, Gottdiener J, et al. Frailty in older adults: evidence for a phenotype. J Gerontol A Biol Sci Med Sci 2001;56(3):M146-56.

[4] Strandberg TE, Pitkala KH. Frailty in elderly people. Lancet 2007;369(9570): $1328-9$.

[5] Fisher AL. Just what defines frailty? J Am Geriatr Soc 2005;53(12):2229-30.

[6] Fried LP, Ferrucci L, Darer J, Williamson JD, Anderson G. Untangling the concepts of disability, frailty, and comorbidity: implications for improved targeting and care. J Gerontol A Biol Sci Med Sci 2004;59(3):255-63.

[7] Morley JE, Haren MT, Rolland Y, Kim MJ. Frailty. Med Clin North Am 2006;90(5):837-47.

[8] Clegg A, Young J, Iliffe S, Rikkert MO, Rockwood K. Frailty in elderly people. Lancet 2013;381(9868):752-62.

[9] Chin A, Paw MJ, van Uffelen JG, Riphagen I, van Mechelen W. The functional effects of physical exercise training in frail older people: a systematic review. Sports Med 2008;38(9):781-93.
[10] Chou CH, Hwang CL, Wu YT. Effect of exercise on physical function, daily living activities, and quality of life in the frail older adults: a meta-analysis. Arch Phys Med Rehabil 2012;93(2):237-44

[11] Theou O, Stathokostas L, Roland KP, Jakobi JM, Patterson C, Vandervoort AA, et al. The effectiveness of exercise interventions for the management of frailty: a systematic review. J Aging Res 2011;2011:569194.

[12] Cadore EL, Rodriguez-Manas L, Sinclair A, Izquierdo M. Effects of different exercise interventions on risk of falls, gait ability, and balance in physically frail older adults: a systematic review. Rejuvenation Res 2013;16(2):10514.

[13] Faber MJ, Bosscher RJ, Chin A, Paw MJ, van Wieringen PC. Effects of exercise programs on falls and mobility in frail and prefrail older adults: a multicenter randomized controlled trial. Arch Phys Med Rehabil 2006;87(7):88596.

[14] Cesari M, Vellas B, Hsu FC, Newman AB, Doss H, King AC, et al. A physical activity intervention to treat the frailty syndrome in older persons-results from the LIFE-P study. J Gerontol A Biol Sci Med Sci 2015;70(2):216-22.

[15] Cameron ID, Fairhall N, Langron C, Lockwood K, Monaghan N, Aggar C, et al. A multifactorial interdisciplinary intervention reduces frailty in older people: randomized trial. BMC Med 2013;11:65 [7015-11-65].

[16] Robertson DA, Savva GM, Kenny RA. Frailty and cognitive impairment - a review of the evidence and causal mechanisms. Ageing Res Rev 2013;12(4):840-51.

[17] Sampson EL. Frailty and dementia: common but complex comorbidities. Aging Ment Health 2012;16(3):269-72.

[18] Gillette-Guyonnet S, Nourhashemi F, Andrieu S, de Glisezinski I, Ousset PJ, Riviere D, et al. Weight loss in Alzheimer disease. Am J Clin Nutr 2000;71(2):637S-42S.

[19] Burns JM, Johnson DK, Watts A, Swerdlow RH, Brooks WM. Reduced lean mass in early Alzheimer disease and its association with brain atrophy. Arch Neurol 2010;67(4):428-33.

[20] Cruz-Jentoft AJ, Landi F, Topinkova E, Michel JP. Understanding sarcopenia as a geriatric syndrome. Curr Opin Clin Nutr Metab Care 2010;13(1):1-7.

[21] Heyn P, Abreu BC, Ottenbacher KJ. The effects of exercise training on elderly persons with cognitive impairment and dementia: a meta-analysis. Arch Phys Med Rehabil 2004;85(10):1694-704.

[22] Pitkala KH, Raivio MM, Laakkonen ML, Tilvis RS, Kautiainen H, Strandberg TE. Exercise rehabilitation on home-dwelling patients with Alzheimer's disease a randomized, controlled trial. Study protocol. Trials 2010;11:92 [6215-1192].

[23] Pitkala KH, Poysti MM, Laakkonen ML, Tilvis RS, Savikko N, Kautiainen H, et al. Effects of the Finnish Alzheimer disease exercise trial (FINALEX): a randomized controlled trial. JAMA Intern Med 2013;173(10):894-901.

[24] Guigoz Y, Lauque S, Vellas BJ. Identifying the elderly at risk for malnutrition. The Mini Nutritional Assessment. Clin Geriatr Med 2002;18(4):737-57.

[25] Charlson ME, Pompei P, Ales KL, MacKenzie CR. A new method of classifying prognostic comorbidity in longitudinal studies: development and validation. J Chronic Dis 1987:40(5):373-83.

[26] Hughes CP, Berg L, Danziger WL, Coben LA, Martin RL. A new clinical scale for the staging of dementia. Br J Psychiatry 1982;140:566-72.

[27] Folstein MF, Folstein SE, McHugh PR. "Mini mental state". A practical method for grading the cognitive state of patients for the clinician. J Psychiatr Res 1975;12(3):189-98.

[28] Pollak N, Rheault W, Stoecker JL. Reliability and validity of the FIM for persons aged 80 years and above from a multilevel continuing care retirement community. Arch Phys Med Rehabil 1996;77(10):1056-61.

[29] Alexopoulos GS, Abrams RC, Young RC, Shamoian CA. Cornell scale for depression in dementia. Biol Psychiatry 1988:23(3):271-84.

[30] Guralnik JM, Simonsick EM, Ferrucci L, Glynn RJ, Berkman LF, Blazer DG, et al. A short physical performance battery assessing lower extremity function: association with self-reported disability and prediction of mortality and nursing home admission. J Gerontol 1994;49(2):M85-94. 